\title{
HACIENDO VISIBLE LO INVISIBLE: VISUALIZACIÓN DE LA ESTRUCTURA DE LAS RELACIONES EN RED EN TWITTER POR MEDIO DEL ANÁLISIS DE REDES SOCIALES
}

\section{Miguel Del-Fresno-García}

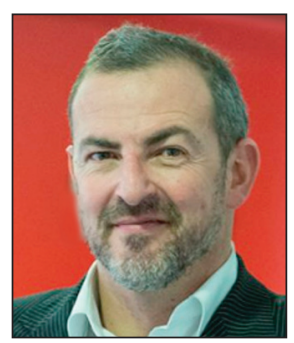

Miguel Del-Fresno-García es profesor en la Universidad Nacional de Educación a Distancia (UNED), doctor en sociología (UNED), DEA y master en sociedad de la información y el conocimiento (UOC), MBA y executive Master en e-business (IE Business School) y licenciado en Filosofía (UCM). Imparte docencia en masters de comunicación en la Universidad del País Vasco (UPV), UCM, Universidad de Sevilla y Universidad Jaume I. Profesor visitante en la Universidad de la República de Uruguay (Montevideo). Investigador visitante en U. C. Berkeley (EUA), U. C. San Diego (EUA). Anteriormente ha sido director de marketing y comunicación y director de unidad de negocio en Elsevier España; director de marketing y comunicación de la cadena de librerías Casa del Libro; director de marketing y miembro del equipo fundador de casadellibro.com. Sus últimos libros sobre investigación social online son El consumidor social y Netnografía.

http://orcid.org/0000-0003-2746-3681

Universidad Nacional de Educación a Distancia Obispo Trejo, s/n. 28040 Madrid, España mdelfresno@der.uned.es

\section{Resumen}

Vivimos inmersos en una densa red social de interacciones que nos conectan a personas, información, eventos y lugares facilitando o restringiendo el flujo de información, ideas y percepciones, en un instantáneo y masivo sistema de comunicación en red. Creamos artefactos cognitivos que integran la visualización de datos y los procesos cognitivos para incrementar nuestras capacidades para comprender la creciente complejidad del mundo externo, al que se han sumado los social media de internet. Se combina Twitter como campo de investigación, y el análisis de redes sociales (ARS) como marco analítico y conjunto de métodos para capturar, analizar y visualizar datos empíricos tanto de forma estática (imágenes representando redes en ciertos puntos del tiempo) como dinámica (vídeo reflejando datos longitudinales). Se presenta Flocker, una herramienta diseñada para la visualización y representación en tiempo real de la actividad en red en Twitter.

\section{Palabras clave}

Visualización de información, Visualización de redes sociales, Análisis de redes rociales, ARS, Twitter, Medios sociales, Flocker.

Title: Making the invisible visible: visualizing the network structure of Twitter relationships using Social

Network Analysis

\begin{abstract}
We live in a dense social network of interactions that connect us to people, information, events and places facilitating or constraining the flow of information, ideas, perceptions, etc., into an instant and massive network communication system. We create cognitive artefacts that integrate data visualization and cognitive processes in order to increase our ability to understand the complexity of the external world, made even more complex with the Internet and rise of social media. In this paper, we combine Twitter, as a type of fieldwork site, and Social Networks Analysis (SNA), as both an analytic framework and set of methods, to capture, analyze, and visualize empirical data, both statically (figure representing point in time) and dynamically (video reflecting longitudinal data). We present Flocker, a tool designed for real-time visualization and representation of network activity using Twitter as an illustrative case.
\end{abstract}

\section{Keywords}

Information visualization, Social network visualization, Social network analysis, SNA, Twitter, Social media, Flocker.

Del-Fresno-García, Miguel (2014). “Haciendo visible lo invisible: visualización de la estructura de las relaciones en red en Twitter por medio del análisis de redes sociales". El profesional de la información, mayo-junio, v. 23, n. 3, pp. $246-252$.

http://dx.doi.org/10.3145/epi.2014.may.04 


\section{Introducción}

La era de la información (Castells, 2001) o sociedad de la información (Mattelart, 2002) se caracteriza por los cambios sociales sucedidos desde las décadas finales del siglo $X X$, derivados de la evolución de las tecnologías digitales de información y comunicación junto con la emergencia de una estructura social en red, que ha afectado a todos los ámbitos de la actividad humana a escala global.

Los cambios sociales producidos por la innovación tecnológica han provocado "variaciones en la ordenación de las relaciones interpersonales" (Del-Fresno-García, 2011a, p. 20) y una creciente tecnologización de la realidad (Virilio, 1991), el mundo externo, con un crecimiento exponencial de los datos y la información disponible hasta convertirse en un problema colectivo: el exceso de información o information overload (Toffler, 1970; Toffler; Toffler, 1994). Para Alvin Toffler el exceso de información desencadena un mecanismo psicológico de defensa que provoca que, individuos y sociedades, tiendan a simplificar el mundo externo y lleva a la selección de aquellos datos que confirman los prejuicios previos, ignorando el resto de los datos existentes. Ello provoca que el acceso, el uso y la comprensión del conocimiento existente sea cada día más difícil.

Si la década de los años 70 del siglo XX, según Paul Krugman, fue el punto más alto en la generación "de grandes cantidades de teoría aplicada desde muy pequeñas cantidades de datos" (Silver, 2013, p. 7), en los inicios de la segunda década del siglo XXI emerge el big data. La promesa implícita del big data es que la solución al exceso de información pasa por mayores cantidades de datos. Lo que se ha denominado como era del petabyte, "un mundo en el que ingentes cantidades de datos y las matemáticas aplicadas reemplazan cualquier otro instrumento", lo que implica que "el volumen de los datos obviará la necesidad de teoría, e incluso del método científico" (Anderson, 2008).

La cognición como proceso de "adquisición o uso del conocimiento" tiene la ventaja de "centrarse tanto en el objeto de visualización como en el medio" (Card; Mackinlay; Shneiderman, 1999, p. 6). El desarrollo de técnicas y métodos para incrementar nuestras capacidades analíticas para el pensamiento y el razonamiento, cognición externa o external cognition (Scaife; Rogers, 1996), es una necesidad ya que los errores pueden ser producidos también de forma masiva. La crisis económica de finales de 2007, en la cual los datos que mostraban los problemas estaban disponibles con antelación pero no se interpretaron de forma correcta, los atentados del 11-S, del 15-M, etc., muestran la dimensión que puede alcanzar una interpretación deficiente de gran cantidad de datos existentes. Así, el big data podría estar ya mostrando dos debilidades significativas:

- una deficiencia metodológica: el foco se pone en los grandes volúmenes de datos y no en el proceso de toma de decisiones correctas;

- ingenuidad epistemológica: el futuro nunca es una prolongación lineal del pasado.

Incluso con las mejores técnicas de big data es imposible distinguir hoy por hoy lo imposible de lo altamente impro- bable. Somos nosotros quienes debemos dotar a los datos de sentido y significado. La demanda debe ser mejores capacidades analíticas para comprender los significados de los datos y no, de forma determinista, más cantidades de datos. Usando la visión para pensar, las técnicas de representación de datos e información permiten crear artefactos cognitivos que posibilitan la integración de la visualización y los procesos cognitivos con el objetivo de amplificar -más y mejor- nuestra comprensión de patrones no identificados en la creciente complejidad del mundo externo. La visualización de forma estática o dinámica de las relaciones, no físicas, basadas las interacciones sociales en los social media de internet, en concreto en Twitter, es el objeto del presente trabajo de investigación.

\section{Social media: nosotros los medios}

Internet es el fenómeno socio-tecnológico más disruptivo para la comunicación, al menos, desde la llegada de la televisión. Las relaciones que se dan en internet y sus social media forman parte destacada de ese mundo externo, se producen de forma masiva y tienden a permanecer invisibles. Capturar y representar esas relaciones nos enfrenta con nuestros límites cognitivos ante la necesidad de comprender la sociabilidad en un contexto sin precedentes como el ciberespacio, "un lugar sin espacio" (Del-Fresno-García, 2011a, p. 19). El resultado es que hoy todo está conectado: personas, información, eventos y lugares, creando relaciones entre sí y formando de manera agregada una vasta red social donde "ya no es ni significativa ni oportuna una separación ontológica ni fenomenológica entre el mundo online y offline" (Del-Fresno-García, 2011a, p. 61) como parte de un mismo continuum social.

\section{Las relaciones que se dan en internet y sus social media se producen de forma masiva y tienden a permanecer invisi- bles}

\subsection{Autocomunicación de masas y micromedios}

Desde el punto de vista social y relacional, internet y su arquitectura han permitido la evolución y uso de plataformas orientadas de manera específica a la interacción personal (Facebook, Twitter, blogs, foros, agregadores de opiniones, medios de comunicación profesionales sólo online, etc.). Desde un punto de vista técnico, los social media son aplicaciones que permiten la creación e intercambio de contenidos generados por los usuarios (Kaplan; Haenlein, 2009) por medio de la interacción, síncrona y asíncrona, con un alcance sin precedentes en la historia de la humanidad. Ello supone un complejo entramado relacional entre prácticas comunicativas y sociales e infraestructura tecnológica dando lugar a un nuevo ecosistema de comunicación interpersonal colectiva (Del-Fresno-García, 2012).

El estadio básico de captura y representación de datos de internet son las técnicas de recuperación de información (information retrieval) por medio de herramientas de monitorización (Astigarraga et al., 2011; Del-Fresno-García, 
2011b; González-Gálvez; Rey-Martín; Cavaller-Reyes, 2011; Sagarminaga-Gutiérrez; Canals, 2011), lo que se puede considerar un press clipping online, que carece, sin embargo, de la posibilidad de mostrar cómo se comparte la información en red entre entidades (individuos, organizaciones, etc.).

A través de un nuevo conjunto de métodos que unen la captura y visualización de las interacciones en los social media y el análisis de redes sociales (ARS) o social network analysis (SNA) es posible representar y analizar esas interacciones como redes con una profundidad y escala nunca antes posible. Una de las plataformas más destacadas por su dinamismo y crecimiento, local y global, es Twitter.

\subsection{Por qué Twitter}

Twitter es un medio social online global y gratuito, sin un claro modelo de negocio definido desde sus inicios, que combina elementos de los blogs, mensajes de texto y emisión de información. Los usuarios escriben mensajes cortos limitados a 140 caracteres, conocidos como tweets, que se envían a todos aquellos que han optado por recibir los tweets del emisor. En cada tweet es posible enlazar o incrustar vídeo, imágenes y hashtags (una palabra o frase prefijada con el símbolo \# que las convierte en metadatos). Aunque puede parecer difícil una comunicación significativa en Twitter, los usuarios "han encontrado formas creativas para conseguir el máximo rendimiento de cada tweet mediante el uso de diferentes herramientas de comunicación" (Lovejoy; Waters; Saxton, 2012, p. 313). En Twitter se puede capturar una extensa variedad de información en tiempo real, y de forma retrospectiva, lo que supone un acceso sin precedentes a los registros de la actividad humana online en el tiempo.

Twitter puede ser definido como un microblogging conversacional y, además de millones de personas, muchas empresas, organizaciones, medios de comunicación, ONGs o gobiernos lo utilizan para fines de comunicación tales como: marketing, atención al cliente, noticias, o incluso, activismo. Dadas sus propiedades aparentemente simples e inherentemente flexibles, en particular la brevedad y la velocidad, Twitter seguirá adaptándose a nuevos usos y usuarios en el futuro a pesar de los pronósticos de los escépticos y es muy probable que siga creciendo impulsado por el crecimiento de las aplicaciones para móviles, para los que Twitter es especialmente adecuado (Arceneaux; Schmitz, 2010) y ser un medio de intersección de every media \& medium (Dorsey, 2012).

El interés de Twitter para esta investigación es doble:

1) permite el intercambio de comunicación de forma masiva e interpersonal que puede ser capturada, almacenada, representada y analizada;

2) los usuarios se expresan al menos por medio de dos tipos de conexiones explícitas: retweets (RTs) y menciones o respuestas (MTs).

Se trata de dos tipos de conexiones explícitas, más relevantes que el indicador común de número de seguidores/ seguidos. MTs y en especial RTs, son actos significativos al demostrarse que "sólo el $6 \%$ de todos los tweets son republicados" (Elmer, 2013, p. 19) y "menos de uno cada 200 tweets son retweeteados después de una hora de ser publicados" (Geere, 2010).

\section{Análisis y visualización de redes sociales online}

Vivimos inmersos en una densa red de interacciones y relaciones sociales que nos unen a otros y facilitan el flujo de información, ideas, percepciones, etc., en un instantáneo y masivo sistema de comunicación, global y local, en red. Como metáfora, el término red o network es muy gráfico, ya que evoca imágenes de nodos (individuos) conectados por medio de líneas (relaciones). Una red social consiste en "un conjunto finito de actores y la relación definida entre esos actores" (Wasserman; Faust, 1994, p. 20) considerados como unidades interdependientes y que pueden ser representadas por medio de un grafo.

La teoría y aplicación del ARS ofrece un conjunto de métodos para el análisis de las interacciones sociales que permiten de forma específica investigar las estructuras relacionales y su representación como redes. Los investigadores en ARS -desde las matemáticas, la física y las ciencias computacionales- han aportado conceptos y métricas aplicables al estudio sistemático de procesos sociales impensables hace unos años para las ciencias sociales. El ARS proporciona tanto acceso a nuevo conocimiento como la representación de las estructuras relacionales y como éstas pueden ser consecuencia de la acción individual y colectiva. Para Linton C. Freeman (1979; 2000) el ARS tiene cuatro características singulares:

a) asume una intuición estructural de las relaciones sociales;

b) la recogida de datos empíricos ha de realizarse de forma sistemática;

c) los modelos matemáticos son una parte fundamental del análisis con la ayuda de ordenadores como herramientas para su explotación y visualización;

d) crear y compartir las visualizaciones de las relaciones, los patrones de las interacciones, permite la generación de insights estructurales significativos y su comunicación a otros.

Su aplicación al contexto de los social media o web 2.0 aún está en fase de desarrollo embrionario (Xu; Lin, 2013; DelFresno-García; Daly, 2014) y es de esperar que se desarrollen más investigaciones empíricas en breve.

Degree o grado es una medida simple que mide el número de conexiones de un nodo y esta relacionado con la influencia y capacidad de difusión en red

\section{Metodología}

Se presentan dos métodos, directo e indirecto, de capturar y extraer los datos relacionales de Twitter a partir de conexiones explícitas (RTs y MTs) para su posterior representación, visualización y análisis de forma estática (imágenes representando redes en ciertos puntos del tiempo) y dinámica (vídeo reflejando datos longitudinales) como redes por medio del ARS. 


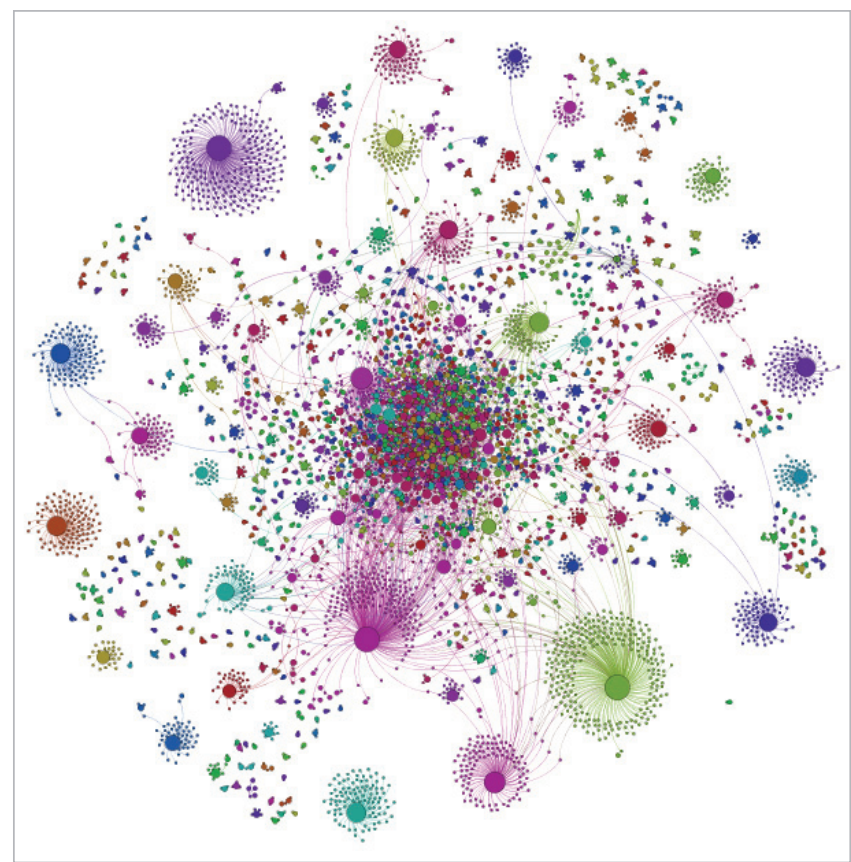

Figura 1. Red de RT con in-degree

El concepto de centralidad en ARS aborda la cuestión de qué nodos son los más importantes o centrales en una red. Degree o grado es una medida simple que mide el número de conexiones de un nodo y esta relacionado con la influencia y capacidad de difusión en red. El degree de un nodo se calcula como la suma de in-degree (grado entrante) y out-degree (grado saliente). El in-degree es el número de conexiones que apuntan hacia un nodo y out-degree es el número de conexiones que se originan en un nodo apuntando hacia otros nodos. En ARS existen numerosos indicadores para interpretar diferentes roles de los nodos en una red. En las imágenes presentadas el tamaño de los nodos está relacionado con su degree o in-degree.

\subsection{Captura directa de datos contra la API de Twitter}

La captura y extracción de datos desde Twitter se realiza directamente contra la API (application programming interface) de Twitter ${ }^{1}$. Tras la definición previa del objeto de estudio por medio de una palabra o frase clave, hashtag, etc., se define el campo de estudio:

- por el número de tweets total que se desean capturar -desde miles a millones-

- por un lapso temporal -desde horas a meses-.

Esta amplitud en volumen de datos y lapso de tiempo sólo es viable con este método.

Tras la captura y extracción se genera un fichero con la totalidad de las interacciones del que se generan dos nuevos ficheros (red de RTs y red de MTs). Los nicknames de los usuarios pueden ser sustituidos con un ID único para garantizar el anonimato. Los ficheros (la red de RTs, la red de MTs y la red total) se generan en formatos importables desde software de $\mathrm{ARS}^{2}$ para su representación, visualización y explotación analítica.

\subsection{Flocker: captura indirecta de datos de Twitter}

Para evitar requerimientos técnicos de programación se ha

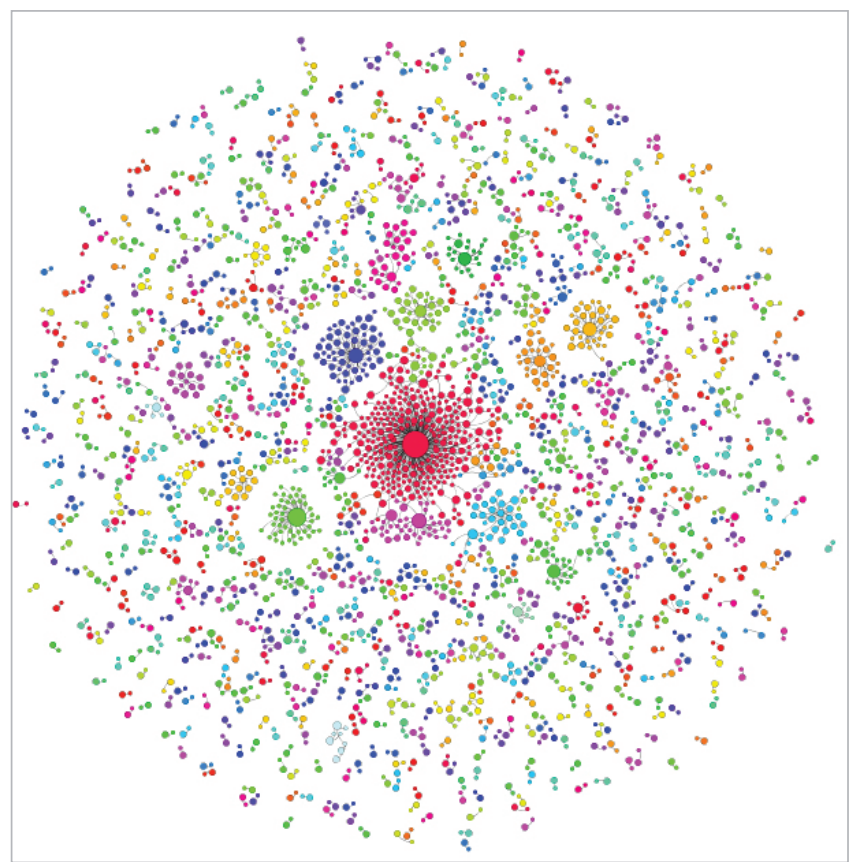

Figura 2. Red de MT con in-degree

desarrollado Flocker ${ }^{3}$, una herramienta online específica para la visualización y representación en tiempo real de la actividad en Twitter. Tras la definición del objeto de estudio por medio de una palabra clave (en el ejemplo: EPA), el campo se define:
- por el número de tweets a capturar
- por el lapso temporal que el usuario deberá definir.

Una vez obtenida la red es posible exportar las imágenes de la red obtenida (.svg o .png) y la propia red (.gexf). Flocker también permite el acceso a datos adicionales: laboratorio de datos, tag cloud de la red y rankings de tweets, usuarios y urls con más RTs.

http://flocker.outliers.es

\section{Resultados}

\subsection{Captura directa de datos y representación en red en imágenes}

La representación de las redes de RT y MTS (figuras 1 y 2):

1) Figura 1. Red RTs: $n=6.230$ nodos y 5.568 conexiones, aplicando el algoritmo de in-degree.

2) Figura 2. Red MTs: $n=3.036$ nodos y 1.999 conexiones, aplicando el algoritmo de in-degree.

3) Figura 3. Red de imágenes (nodos en rojo) más compartidas entre usuarios (nodos en azul) de una red por medio de RTs: $n=4.514$ nodos y 6.040 conexiones, aplicando el algoritmo de in-degree.

\subsection{Captura indirecta de datos y representación en red en imágenes}

Para la captura y representación de las redes con Flocker (figura 3) y Gephi" (figura 4) con la keyword de búsqueda "EPA" de un total de 9.976 tweets genera la siguiente red.

1) Figuras 4 y 5 . Red: $n=5.248$ nodos y 6.212 conexiones, aplicando el algoritmo de in-degree. 


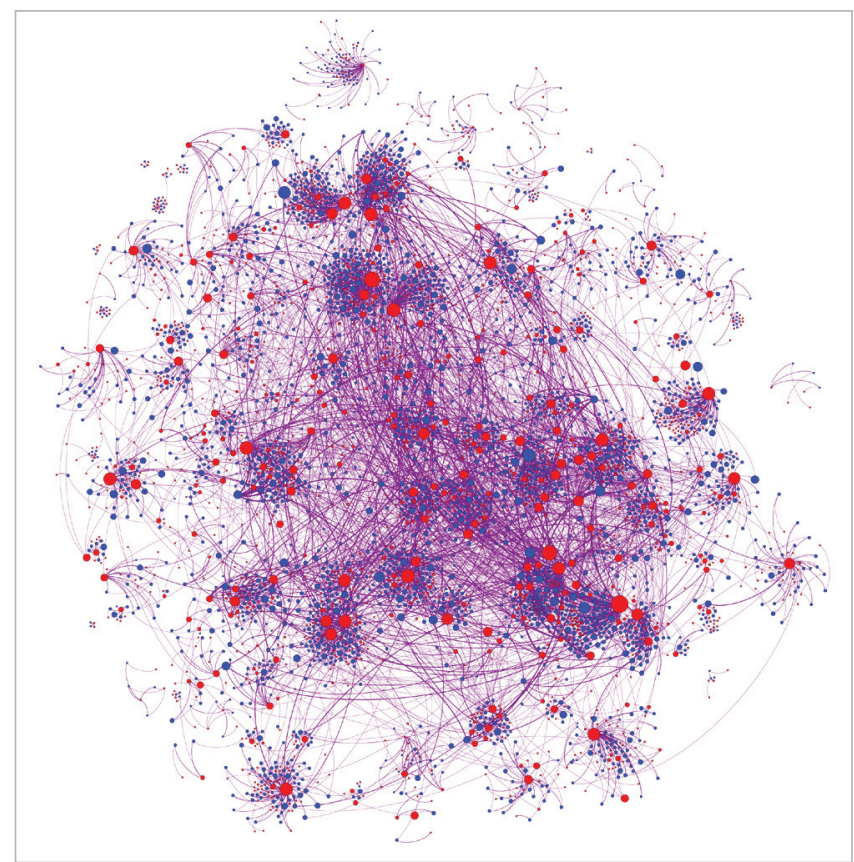

Figura 3. Red usuarios (azul) e imágenes (rojo) como más RTs con in-degree

\subsection{Captura directa de datos y representación en ví- deo}

A partir de los datos del apartado 5.1 y utilizando el método propuesto por Esteban Moro (2012) se generaron dos vídeos de la red completa presentada en 5.1:

- red de tweets instantánea: SNA ARS instantaneous 1 https://www.youtube.com/watch?v=ofY9OG-LKQI

- red de tweets agregada: SNA ARS aggregated 2 https://www.youtube.com/watch?v=suz1YoC5Qek

\subsection{Captura indirecta de datos y representación en vídeo}

Utilizando Flocker y la keyword de búsqueda "New York" se capturaron 5.000 tweets, y con software de captura de pantalla se generó un vídeo (velocidad 8X) de la creación de la red y sus relaciones: New York around 5.000 tweets https://www.youtube.com/watch?v=rUkkg-YHi2O

\section{Discusión}

Gracias a la creciente capacidad computacional, el desarrollo de tecnologías específicas y la arquitectura de internet es posible la captura, representación y análisis de los datos relacionales online, con el objetivo de amplificar nuestra cognición sobre el mundo externo que son internet y sus social media. Las redes sociales de las que formamos parte juegan un papel central en nuestras actividades y despliegue social. Visualizar y comprender la influencia de estos patrones de interacción y su relación con una amplia variedad de comportamientos es algo clave para diferentes campos del conocimiento. El ARS aplicado a los social media de internet, en este caso a Twitter, permite hacer visible lo que hasta hace poco era impensable visualizar: la estructura de las relaciones en redes en el ciberespacio. Una de las aplicaciones prácticas directas del ARS a diversos ámbitos de la comunicación es la identificación de nuevos tipos de social media influencers (Del-Fresno-García; Daly, 2014)

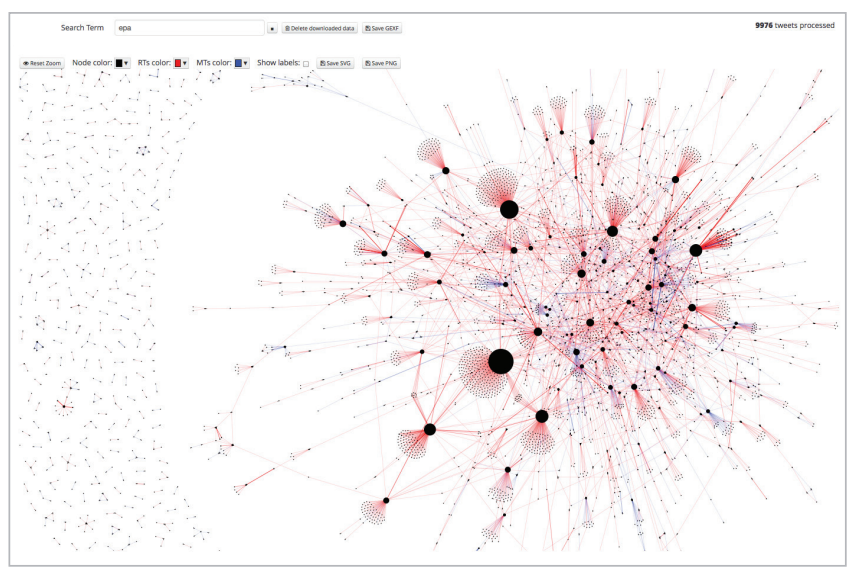

Figura 4. Red de tweets con in-degree (Flocker)

El ARS aplicado a este nuevo ámbito como la sociabilidad online facilita además de lo ya mencionado:

- identificación de individuos clave en esas redes;

- segmentación e identificación de grupos, cliques y comunidades;

- seguimiento de los comportamientos de los miembros de la red;

- cambios en las redes en una doble vertiente: cómo el despliegue social de los individuos modifica la estructura de la red y cómo la estructura de la red condiciona las posibilidades de sus miembros.

Ello abre nuevas vías de investigación sobre formas emergentes de desigualdad en red (Del-Fresno-García, 2014) y la posición, central o periférica, que ocupamos en la misma.

Se ha presentado un método intuitivo y empírico para la representación y visualización de la estructura de las redes y nodos clave en Twitter. No obstante, existen limitaciones ya que la representación de la estructura y relaciones presenta un déficit: la no comprensión de los significados que

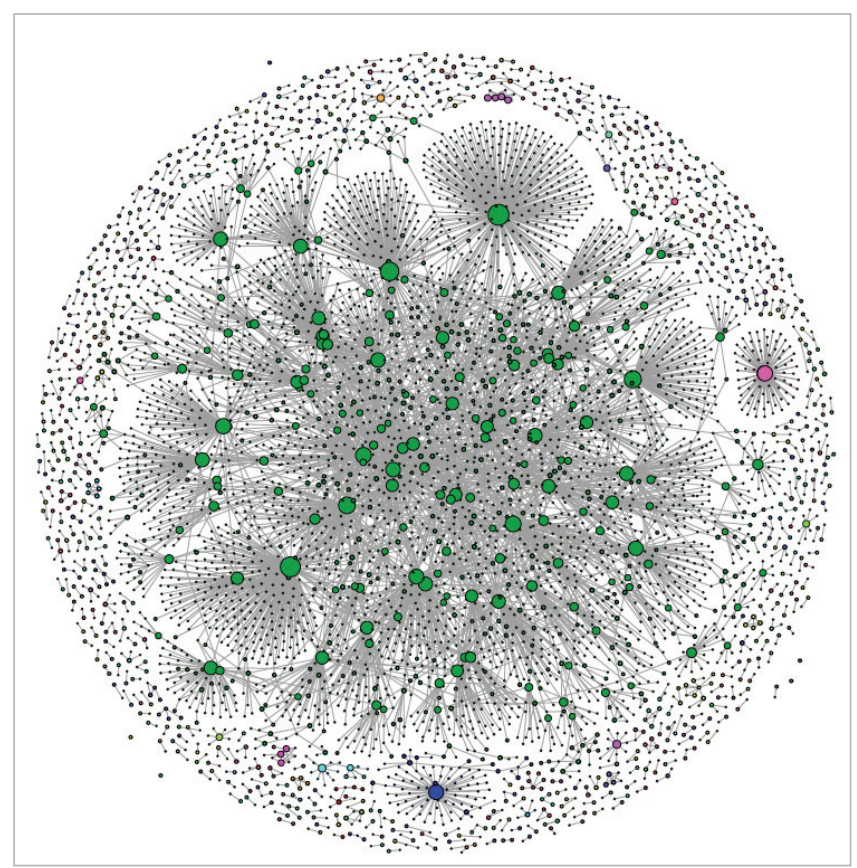

Figura 5. Red de tweets in-degree en Gephi 
circulan por la red y las motivaciones de los individuos que interactúan con otros. Mientras que el ARS proporciona una manera de medir con precisión la cantidad de relaciones, no aporta una explicación cualitativa alrededor de los intercambios que se producen en red. Nuevas investigaciones y metodologías deben incorporar ambas aproximaciones. Cabe destacar la network ethnography (Howard, 2002) o la netnografía (Del-Fresno-García, 2011b; Del-Fresno-García; López, 2014), un enfoque interdisciplinario entre la sociología, antropología, lingüística y comunicación para representar y comprender la creciente complejidad, local y global, de la comunicación y sociabilidad en red.

EI ARS puede ser aplicado a distintas disciplinas desde los siguientes enfoques:

a) la identificación de individuos clave a la hora de promover la difusión de cambios de comportamiento en redes;

b) la segmentación e identificación de grupos, cliques y comunidades;

c) el cambio de comportamientos o adopción de innovaciones a través de los miembros internos de la red y no líderes jerárquicos;

d) la influencia en el seno de las redes puede ser bidireccional: individuos-red y red-individuos.

Ello que abre un amplio campo de exploración a diferentes disciplinas a la hora de identificar individuos y roles clave en el seno de las múltiples redes de las que formamos parte.

\section{Notas}

1. El servidor de Flocker (Python) establece una comunicación con el API de Twitter (vía autorización OAuth) usando las credenciales de un usuario. Gestiona la entrada de datos del streaming de Twitter y genera otro streaming con el navegador del usuario. Este proceso aísla al usuario de la complejidad técnica con la API Twitter. El javascript en la parte cliente (navegador) recibe y procesa los tweets y genera y visualiza la red con D3.js. El factor limitador en el proceso es el propio sistema del usuario.

2. Para un listado actualizado de los programas disponibles http://en.wikipedia.org/wiki/Social_network_analysis_ software

3. Flocker ha sido desarrollado por Outliers. http://outliers.es

4. Gephi es un software gratuito de código abierto para la visualización interactiva, exploración y análisis de grafos de redes.

https://gephi.org

\section{Bibliografía}

Anderson, Chris (2008). "The end of theory: the data deluge makes the scientific method obsolete". Wired, Jun 23.

http://archive.wired.com/science/discoveries/ magazine/16-07/pb_theory

Arceneaux, Noah; Schmitz, Amy (2010). "Seems stupid until you try it: press coverage of Twitter, 2006-9". New media \& society, v. 12 , n. 8 , pp. 1262-1279.
http://mc7290.bgsu.wikispaces.net/file/view/Arceneaux Noah.pdf

http://dx.doi.org/10.1177/1461444809360773

Astigarraga, Eneko; Azpillaga, Josu; Fernández, Luis; Naberan, Aitzol (2011). "Umap, inteligencia colectiva extraída de las redes sociales". El profesional de la información, v. 20, n. 5, pp. 542-547.

http://eprints.rclis.org/16177

http://dx.doi.org/10.3145/epi.2011.sep.08

Card, Stuart; Mackinlay, Jock; Shneiderman, Ben (1999). Readings in information visualization. Using vision to think. San Francisco: Academic Press, 712 pp. ISBN: 978 1558605336

Castells, Manuel (2001). La era de la información: economía, sociedad y cultura. Madrid: Alianza editorial, 645 pp. ISBN: 9788420647401

Del-Fresno-García, Miguel (2011a). Netnografía. Barcelona: UOC, 112 pp. ISBN: 9788497883856

Del-Fresno-García, Miguel (2011b). “Infosociabilidad monitorización e investigación en la web 2.0 para la toma de decisiones". El profesional de la información, v. 20, n. 5, pp. 548-554.

http://eprints.rclis.org/16150

http://dx.doi.org/10.3145/epi.2011.sep.09

Del-Fresno-García, Miguel (2012). “Comprendiendo los social media y mass media: un modelo para el estudio de la comunicación interpersonal colectiva en tiempos de internet". Derecom, n. 11, pp. 99-109.

http://derecom.com/numeros/pdf/delfresno.pdf

Del-Fresno-García, Miguel (2014). “Connecting the disconnected: social work and social network analysis. A methodological approach for identifying within network peer leaders". Arbor, 2014 (en prensa).

Del-Fresno-García, Miguel; López, Antonio (2014). "Social work and netnography: the case of Spain and generic drugs". Qualitative social work, v. 13, n. 1, pp. 85-107. http://migueldelfresno.com/2014/02/netnography-socialwork-the-case-of-spain-and-generic-drugs.html http://dx.doi.org/10.1177/1473325013507736

Dorsey, Jack (2012). "Twitter takes the pulse of the planet. It's the intersection of every media \& médium". Twitter, November 15.

https://twitter.com/TwitterAds/status/269129576318386177

Elmer, Greg (2013). "Live research: twittering an election debate". New media \& society, v. 15, n. 1, pp. 18-30. http://dx.doi.org/10.1177/1461444812457328

Freeman, Linton (1979). "Centrality in social networks: conceptual clarification". Social networks, v. 1, pp. 215-239. http://moreno.ss.uci.edu/27.pdf

Freeman, Linton (2000). "Visualizing social networks". Journal of social structure, v. 1, n. 1.

http://www.bebr.ufl.edu/files/Visualizing\%20social\%20 networks_0.pdf

Geere, Duncan (2010). "It's not just you: 71 percent of 
tweets are ignored". Wired, November 11.

http://www.wired.com/2010/10/its-not-just-you-71percent-of-tweets-are-ignored+

González-Gálvez, Pilar; Rey-Martín, Carina; Cavaller-Reyes, Víctor (2011). "Redes sociales para la inteligencia competitiva. Propuesta de un índice sintético". El profesional de la información, v. 20, n. 5, pp. 525-532.

http://dx.doi.org/10.3145/epi.2011.sep.06

Howard, Philip (2002). "Network ethnography and the hypermedia organization: new media, new organizations, new methods". New media \& society, v. 4, n. 4, pp. 550-574.

http://dx.doi.org/10.1177/146144402321466813

Kaplan, Andreas; Haenlein, Michael (2010). "Users of the world, unite! The challenges and opportunities of social media", Business horizons, v. 53, n. 1, pp. 59-68.

http://dx.doi.org/10.1016/j.bushor.2009.09.003

Lovejoy, Kristen; Waters, Richard; Saxton, Gregory (2012). "Engaging stakeholders through Twitter: how nonprofit organizations are getting more out of 140 characters or less". Public relations review, v. 38, p. 313-318.

http://arxiv.org/pdf/1106.1852.pdf

http://dx.doi.org/10.1016/j.pubrev.2012.01.005

Mattelart, Armand (2002). Historia de la sociedad de la información. Barcelona: Paidós, 193 pp. ISBN: 97884493 20422

Moro, Esteban (2012). "Temporal networks with igraph and R (with 20 lines of code!)". Implicit none, November 11.

http://www.r-bloggers.com/temporal-networks-withigraph-and-r-with-20-lines-of-code

Sagarminaga-Gutiérrez, Naiara; Canals, Agustí (2011). “Re- cuperación y tratamiento de opinión movilizada para el análisis del clima social". El profesional de la información, v. 20, n. 5, pp. 510-515.

http://eprints.rclis.org/16153

http://dx.doi.org/10.3145/epi.2011.sep.04

Scaife, Mike; Rogers, Yvonne (1996). “External cognition: how do graphical representations work?". International journal of human-computer studies, v. 45, n. 2, pp. 185-213. http://users.mct.open.ac.uk/yvonne.rogers/papers/ externalcognition.pdf

http://dx.doi.org/10.1006/ijhc.1996.0048

Silver, Nate (2013). The signal and the noise: why so many predictions fail - but some don't. New York: Penguin Books, 544 pp. ISBN: 9781594204111

Toffler, Alvin (1970). Future shock. New York: Bantam Books, 286 pp. ISBN: 9780553277371

http://cdn.preterhuman.net/texts/literature/general/ Alvin\%20Toffler\%20-\%20Future\%20Shock.pdf

Toffler, Alvin; Toffler, Heidi (1994). "Surfing the third wave". Micro times, n. 118, January 3.

http://mdelf.es/ $x \times 4 n$

Virilio, Paul (1991). The aesthetics of disappearance. New York: Semiotext(e), 128 pp. ISBN: 9781570270413

Wasserman, Stanley; Faust, Katherine (1994). Social network analysis: methods and applications. New York and Cambridge: Cambridge University Press, 857 pp. ISBN: 978 0521387071

Xu, Guandong; Lin, Li (2013). Social media mining and social network analysis: emerging research. IGI Global, 347 pp. ISBN: 9781466628069

\title{
Anuario ThinkEPI 2014
}

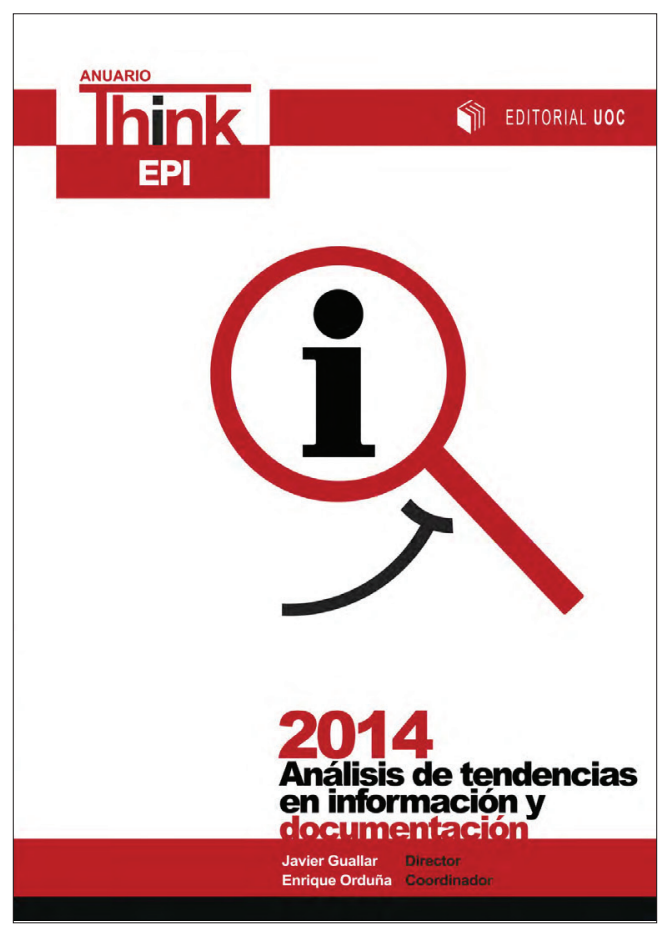

\section{4 páginas de análisis de tendencias en información, documentación y comunicación}

\author{
Formulario de compra: \\ http://www. \\ elprofesionaldelainformacion.com/ \\ suscripciones.php
}

\section{Información y pedidos:} Isabel Olea

epi.iolea@gmail.com

+34608491521 